\title{
Quantifying Electron Correlation of the Chemical Bond
}

DOI:

10.1021/acs.jpclett.7b00535

\section{Document Version}

Accepted author manuscript

Link to publication record in Manchester Research Explorer

\section{Citation for published version (APA):}

Mcdonagh, J., Silva, A. F., Vincent, M., \& Popelier, P. (2017). Quantifying Electron Correlation of the Chemical Bond. Journal of Physical Chemistry Letters, 8(9), 1937-1942. https://doi.org/10.1021/acs.jpclett.7b00535

\section{Published in:}

Journal of Physical Chemistry Letters

\section{Citing this paper}

Please note that where the full-text provided on Manchester Research Explorer is the Author Accepted Manuscript or Proof version this may differ from the final Published version. If citing, it is advised that you check and use the publisher's definitive version.

\section{General rights}

Copyright and moral rights for the publications made accessible in the Research Explorer are retained by the authors and/or other copyright owners and it is a condition of accessing publications that users recognise and abide by the legal requirements associated with these rights.

\section{Takedown policy}

If you believe that this document breaches copyright please refer to the University of Manchester's Takedown Procedures [http://man.ac.uk/04Y6Bo] or contact uml.scholarlycommunications@manchester.ac.uk providing relevant details, so we can investigate your claim.

\section{OPEN ACCESS}


This document is confidential and is proprietary to the American Chemical Society and its authors. Do not copy or disclose without written permission. If you have received this item in error, notify the sender and delete all copies.

\section{Quantifying Electron Correlation of the Chemical Bond}

\begin{tabular}{|r|l|}
\hline Journal: & The Journal of Physical Chemistry Letters \\
\hline Manuscript ID & jz-2017-00535n.R2 \\
\hline Manuscript Type: & Letter \\
\hline Date Submitted by the Author: & n/a \\
\hline Complete List of Authors: & $\begin{array}{l}\text { McDonagh, James; University of Manchester,UK, Manchester Institute of } \\
\text { Biotechnology } \\
\text { Silva, Arnaldo; Manchester Institute of Biotechnology } \\
\text { Vincent, Mark; University of Manchester, School of Chemistry } \\
\text { Popelier, Paul; MIB, and School of Chemistry }\end{array}$ \\
\hline
\end{tabular}

SCHOLARONE
Manuscripts 


1
2
3
4
5
6
7
8
9
10
11
12
13
14
15
16
17
18
19
20
21
22
23
24
25
26
27
28
29
30
31
32
33
34
35
36
37
38
39
40
41
42
43
44
45
46
47
48
49
50
51
52
53
54
55
56
57
58
60

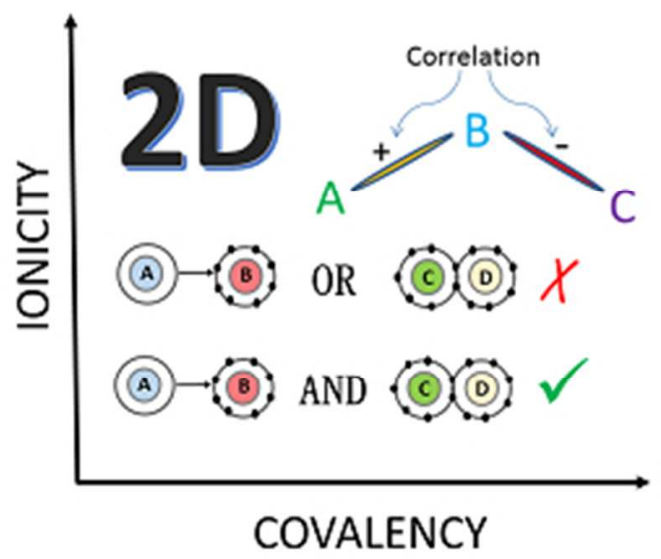

Ionicity and covalency are not each other's opposite. Secondly, bond electron correlation can be destabilising (i.e. positive).

66×56mm (96 x 96 DPI) 


\title{
Quantifying Electron Correlation of the Chemical Bond
}

\author{
James L. McDonagh, Arnaldo F. Silva, Mark A. Vincent, Paul L. A. Popelier* \\ Manchester Institute of Biotechnology (MIB), 131 Princess Street, Manchester M1 7DN, Great Britain and \\ School of Chemistry, University of Manchester, Oxford Road, Manchester M13 9PL, Great Britain
}

\begin{abstract}
The Interacting Quantum Atoms (IQA) method is used to analyze the correlated part of the Møller-Plesset (MP) perturbation theory two-particle density-matrix. Such an analysis determines the effects of electron correlation within atoms and between atoms, which covers both bonds and non-bonded through-space atom-atom interactions. Electron correlation lowers the energy of the atoms at either end of a bond, but for the bond itself, it can be stabilizing or destabilizing. Bonds are described in a twodimensional world of exchange and charge transfer, where covalency is not the opposite of ionicity.
\end{abstract}

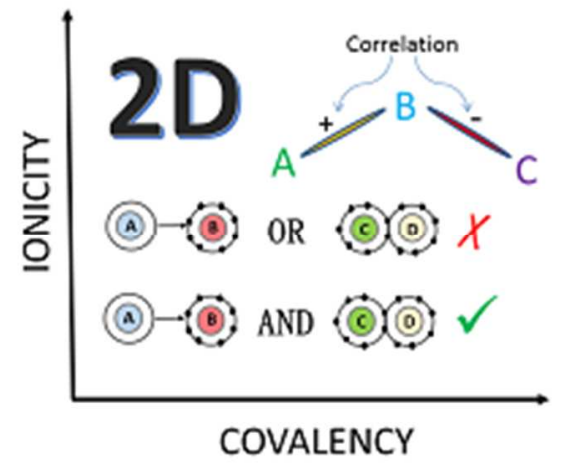

In the traditional view, electron correlation energy is the vitally important component of both inter-atomic and intermolecular energy that binds chemical systems together in the condensed phase. In the case of liquid and solid inert gases it is the only energy binding the atoms together. However, the literature rarely contains a consideration of the intra-molecular energy component of correlation energy. This energy contribution is pivotal for computational methods to improve the description of atoms and bonds within a molecule. Our ultimate interest in correlation energy is to provide the dispersion part of a next generation force field ${ }^{1,2}$ for describing large bio-molecules. However, it is important that we understand how electron correlation affects all aspects of molecules and molecular complexes, not just the through-space long-range parts, if we are to develop an accurate force field for describing matter and its interactions.
Dispersion energy (one component of van der Waals interactions) is often a major energy component arising from electron correlation energy. Oddly, the intramolecular dispersion energy has received little attention in the literature compared to its long-range inter-molecular counterpart. While intra-molecular dispersion energy is included in Møller-Plesset (MP) perturbation calculations, it has not been regularly determined. A commonly applied theoretical tool to obtain through-space non-covalent (dispersive) interactions between two separate molecules is Symmetry Adapted Perturbation Theory (SAPT) ${ }^{3}$. Noncovalent interactions within SAPT are only defined between these fragments as opposed to additionally within a fragment. Hence, a SAPT model of the dispersion interaction between the two termini of a U-shaped chainlike molecule would experience a severe conceptual problem. In this not uncommon case, there is no obvious way to split a single covalently bound molecule (i.e. the chain) into two fragments such that SAPT would be able to 
calculate the dispersion interaction. However, if one abandons SAPT, as we do here, then the conceptual distinction between intra- and inter-molecular interaction disappears. In the current contribution, we will work with topological atoms ${ }^{4}$, which naturally appear in the electron density, and we will calculate their dispersion interaction, wherever they reside. This means that both inter-molecular and intra-molecular dispersion is determined.

Topological atoms are defined by the Quantum Theory of Atoms in Molecules (QTAIM) ${ }^{5-7}$. They are obtained in a parameter-free way and arise naturally out of the electron density: a set of trajectories traced by the gradient of the electron density divide a molecule or molecular complex into topological atoms. All approaches that use the central idea of partitioning a quantum mechanical property density into a set of finite-volume fragments in real 3D space are bundled under the name Quantum Chemical Topology $(\mathrm{QCT})^{8-10}$.

The inclusion of dispersion energy, which is missing or only partially included in Kohn-Sham DFT, is an active area of research for the DFT community ${ }^{11-13}$. A popular approach to include dispersion energy in a tractable QM method is to augment DFT with empirical dispersion corrections ${ }^{14-16}$. While many useful studies have been carried out ${ }^{17,18}$ with such methods, many of which suffer from the problem of needing a damping function to stop the atoms close to each other returning unrealistically large dispersion energies. In the current work, the need for damping functions is completely eliminated due to the use of topological atoms, which are space-filling ${ }^{2}$. Our approach is to obtain dispersion interactions of these topological atoms from the Møller-Plesset perturbation theory (MPx) correction to the Hartree-Fock wavefunction $^{19}$, and thus circumvent the drawbacks that damping functions pose. An additional strength of our approach is that it is not limited to long-range, indeed, the interaction of an atom with itself or an atom directly bonded to it can be calculated. Put more precisely, the electron correlation between electrons within the same (topological) atom is accessible. The resulting values constitute the atomic self-energy, which also includes the atomic kinetic energy, the intra-atomic Coulomb and exchange energy. The proposed method involves the correlated part of the two-particle density-matrix (2PDM), arising from a second order wavefunction (MP2), being partitioned via the Interacting Quantum Atoms (IQA) approach $^{20}$. This gives the correlation energy contributions for the intra-atomic (i.e. a single atom) case and interatomic (i.e. atom-atom) case, where the atoms may or may not be bonded. Thus it is possible to determine the dispersion energy between two non-bonded atoms, and more remarkably, between two bonded atoms. To try and understand the nature of these effects for bonded and nonbonded atoms we have undertaken the current work.

In our approach we take the correlated part of the 2PDM as generated from an MP2 wavefunction by GAUSSIAN09 (G09) ${ }^{21}$ (Link1111 and associated routines) and transform it to the primitive basis. This information is then passed to a locally written program called MORPHY along with the geometry and orbitals (via the standard G09 written WFN file). The IQA analysis defines each atom from the topology of the electron density and then calculates the energy of each of these atoms and the interaction between them. The details of our approach have been given elsewhere ${ }^{19}$, but here we repeat one key equation (eq.1),

$$
\begin{aligned}
V_{e e, c o r r}^{A B}= & \sum_{j=1}^{N_{G}} \sum_{k=1}^{j} k_{j k} \sum_{l=1}^{N_{G}} \sum_{m=1}^{l} k_{l m} d_{j k l m}^{\text {corr, prim }} \times \\
& \int_{\Omega_{A}} d \mathbf{r}_{1} G_{j k}\left(\mathbf{r}_{1}-\mathbf{R}_{j k}\right) \int_{\Omega_{B}} d \mathbf{r}_{2} \frac{1}{r_{12}} G_{l m}\left(\mathbf{r}_{2}-\mathbf{R}_{l m}\right)
\end{aligned}
$$

where $N_{G}$ is the number of primitive Gaussian basis functions ("primitives" in short) and $\Omega$ is the volume of a topological atom. Note that the two $3 \mathrm{D}$ volume integrals in eq.(1) are coupled because the inter-electronic distance $r_{12}$ depends both on $\mathbf{r}_{1}$ and $\mathbf{r}_{2}$. The interaction energy (V) of each atom with itself $(\mathrm{A}=\mathrm{B})$ or with one of the other atoms $(\mathrm{A} \neq \mathrm{B})$ is obtained from the $2 \mathrm{PDM}(d)$ via a $6 \mathrm{D}$ integration.

As MP2 only correlates electron-electron interactions the one-electron terms have their unperturbed Hartree-Fock values. For nearly all cases considered here we used a grid that reproduced the G09-determined correlation energy to about $4 \mathrm{kJmol}^{-1}$. Any exceptions to this will be noted in the tabulated errors given for all systems in the Supporting Information (SI). The basis set employed to determine the energy and geometry was typically $6-31 \mathrm{G}(\mathrm{d}, \mathrm{p})$, with the exceptions given in the Tables S1 and S2 of the SI. A total 56 molecular systems are reported here, covering covalent and ionic bonds, as well as hydrogen bonds, van der Waals complexes, radicals and ions.

The first case we discuss is that of water. The breakdown of the energy contributions of each atom and 
between pairs of atoms is given diagrammatically in Fig. 1. Note that the $\log$ scale is the $\log$ of the absolute value multiplied by the sign of the original energy value. The kinetic energy is positive for each atom, but the corresponding Coulomb, exchange and correlation energies are negative. We next consider the inter-atomic terms (i.e. $\mathrm{O}-\mathrm{H}$ and $\mathrm{H}^{\cdots} \mathrm{H}$ ) and note that they are smaller, not unexpectedly, than the intra-atomic ones. Kinetic energy is manifestly mono-atomic and hence strictly intra-atomic in nature. Note that the correlation energy of the $\mathrm{O}-\mathrm{H}$ bond is positive. Our previous publication ${ }^{19}$ also noted that $\mathrm{H}_{2}$ had a positive bond correlation energy. This positive sign is perhaps surprising given the generally stabilizing (and hence negative) nature of correlation ${ }^{22}$, which is the case for the atoms at either end of the O-H bond. It seems that Hartree-Fock theory overestimates the bond energy, a deficiency that is corrected by electron correlation.

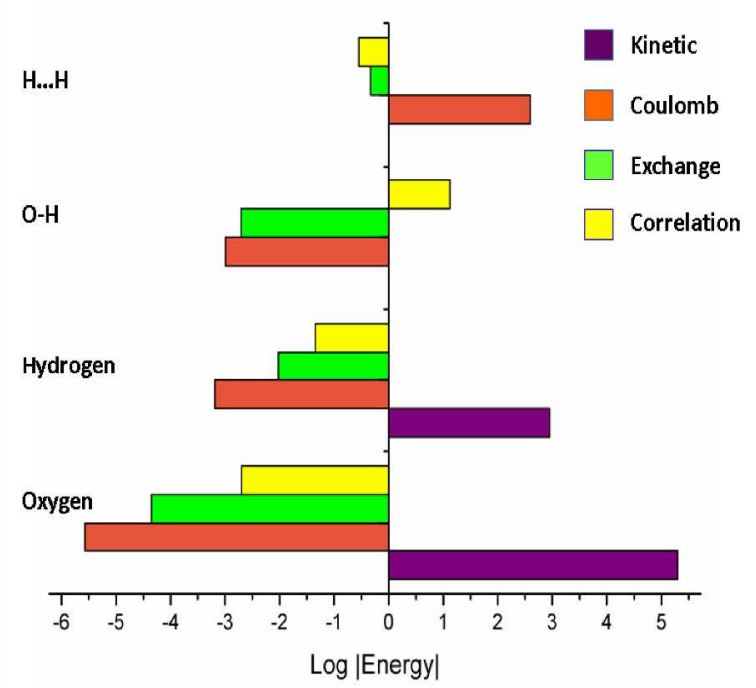

FIG 1. The complete IQA fingerprint of the water molecule. The error of the total energy reconstruction is 1.9 $\mathrm{kJmol}^{-1}$. Negative correlation energies appear on the left; for example, oxygen's Coulomb energy is about $316,000 \approx$ $-10^{5.5} \mathrm{kJmol}^{-1}$.

Thus one's preconceived ideas of correlation are overall correct, but detailed analysis has thrown up a surprise. The last interaction (Fig. 1) is the through-space $\mathrm{H}^{\cdots *} \mathrm{H}$ interaction, which is dominated by a positive Coulomb term. For this interaction the correlation correction is larger than the exchange contribution. In this case the correlation energy is negative. In summary, the energy of a molecule mainly consists of the energy of its constituent atoms and inter-atomic correlation energy can be positive or negative.

All the information described above is very clearly summarized in just one image (Fig.1), which is a complete description of the stability of the water molecule. The full IQA description is unique for each molecule, like a fingerprint. Even though a few common patterns can be observed in similar molecules, some drastic changes can be seen when two molecular fingerprints (such as water and methane) are put side to side. These differences can be seen in the fingerprints of several molecules, presented in the SI (Figures S1 to S11, fingerprint diagrams for some of the molecules studied in the main text: $\mathrm{BeH}_{2}, \mathrm{BH}_{3}, \mathrm{BH}_{4}^{-},\left(\mathrm{BH}_{4}^{-}\right.$ - $\left.\mathrm{BH}_{3}\right), \mathrm{CH}_{4}, \mathrm{NH}_{3}, \mathrm{NH}_{4}^{+},\left(\mathrm{NH}_{4}{ }^{+}-\mathrm{NH}_{3}\right), \mathrm{LiH}, \mathrm{NaH}$ and $\mathrm{KH}$, respectively). Of course, kinetic energy is always positive and exchange energy always negative. A number of more specific observations can be made. Firstly, kinetic energies manifestly destabilize any molecule, but more interestingly, they are the only destabilizing contributions in the triplet $\mathrm{LiH}, \mathrm{NaH}$ and KH. Secondly, the through-space H...H interatomic Coulomb energy is also always destabilizing (in all $\mathrm{AH}_{\mathrm{n}}$ systems studied here, not just the subset in the SI Figures). Thirdly, all A-H Coulomb energies are stabilizing except for $\mathrm{C}-\mathrm{H}$ in methane (Fig.S5). Fourthly, the only other energy contribution that can be destabilizing is A-H correlation, but only in the case of $\mathrm{A}=\mathrm{C}, \mathrm{N}$ or O. Fifthly, lithium's atomic correlation basically vanishes in $\mathrm{LiH}$, which has a very small Li-H bond correlation. However, sodium has a reasonably large atomic correlation in $\mathrm{NaH}$, but its $\mathrm{Na}-\mathrm{H}$ bond correlation is basically zero. Final observations focus on the $\mathrm{BH}_{3} / \mathrm{BH}_{4}^{-}$(Fig. S4) comparison and the $\mathrm{NH}_{3} / \mathrm{NH}_{4}^{+}$comparison (Fig. S8). Energy differences can range from a few $\mathrm{kJmol}^{-1}$ to a few hundred and their patterns are unfortunately too complex to summarize.

Next we considered a wide variety of systems with the aim to understand the nature of bond correlation energies. These systems include radicals and ions with atoms from the $1^{\text {st }}, 2^{\text {nd }}, 3^{\text {rd }}$ and even $4^{\text {th }}$ period. The chosen systems include many $\mathrm{H}-\mathrm{X}$ bonds but also encompass diatomic species and oxides. We have also considered systems such as $\mathrm{He}_{2}$ and the $\mathrm{H}_{2}$ dimer as they are bonded by dispersion and, in the case of the $\mathrm{H}_{2}$ dimer, an additional quadrupolequadrupole interaction ${ }^{23}$. Molecular complexes such as $\mathrm{HF} \ldots \mathrm{F}_{2}$, the $\mathrm{H}_{2} \mathrm{O}$ dimer, the $\mathrm{HF}$ dimer, $\mathrm{H}_{2} \mathrm{O} \ldots \mathrm{NH}_{3}$ and $\mathrm{HF} \ldots \mathrm{H}_{2} \mathrm{O}$ were studied as examples of halogen and 
hydrogen bonds. Thus these systems offer a variety of bonding scenarios with which to gain insight into bond correlation energies. The results for these systems are given diagrammatically in Figures 2, 3 and 4. Note that Figures S13 and S14 provide 3D plots of internuclear distance versus $\left|V_{x}\right|$ and $\left|V_{c}\right|$ for single molecules and complexes, respectively.

Figure 2 plots the absolute value of bond correlation energy against the absolute value of bond exchange energy. The negative bond correlation energies are marked in purple. There is a reasonable correlation between bond correlation and exchange, with a $r^{2}$ value of 0.82 . This correlation expresses itself as a continuous diagonal broad band involving all data points. The same broad correlation can be seen in Figure S12, which focuses on molecular complexes. The points are not randomly distributed in Fig.2 because all ionic molecules are located in the bottom left quadrant, and the apolar covalent molecules are only found in the upper right quadrant. Therefore Fig. 2 can be seen as a diagram in which molecules are distributed according to bond polarity. The central part of the graph is occupied by highly polar covalent bonds such as $\mathrm{H}_{2} \mathrm{O}, \mathrm{HF}$ and $\mathrm{NH}_{3}$. Note that species such as $\mathrm{PH}_{3}, \mathrm{BH}_{3}$ and $\mathrm{SiH}_{4}$ are found the middle of Fig 2. Chemical intuition would refer to those species as covalent with a low bond polarity. However, bonds such as $\mathrm{P}-\mathrm{H}, \mathrm{B}-\mathrm{H}$ and $\mathrm{Si}-\mathrm{H}$ are actually more polar than chemical intuition or an electronegativity argument imply.

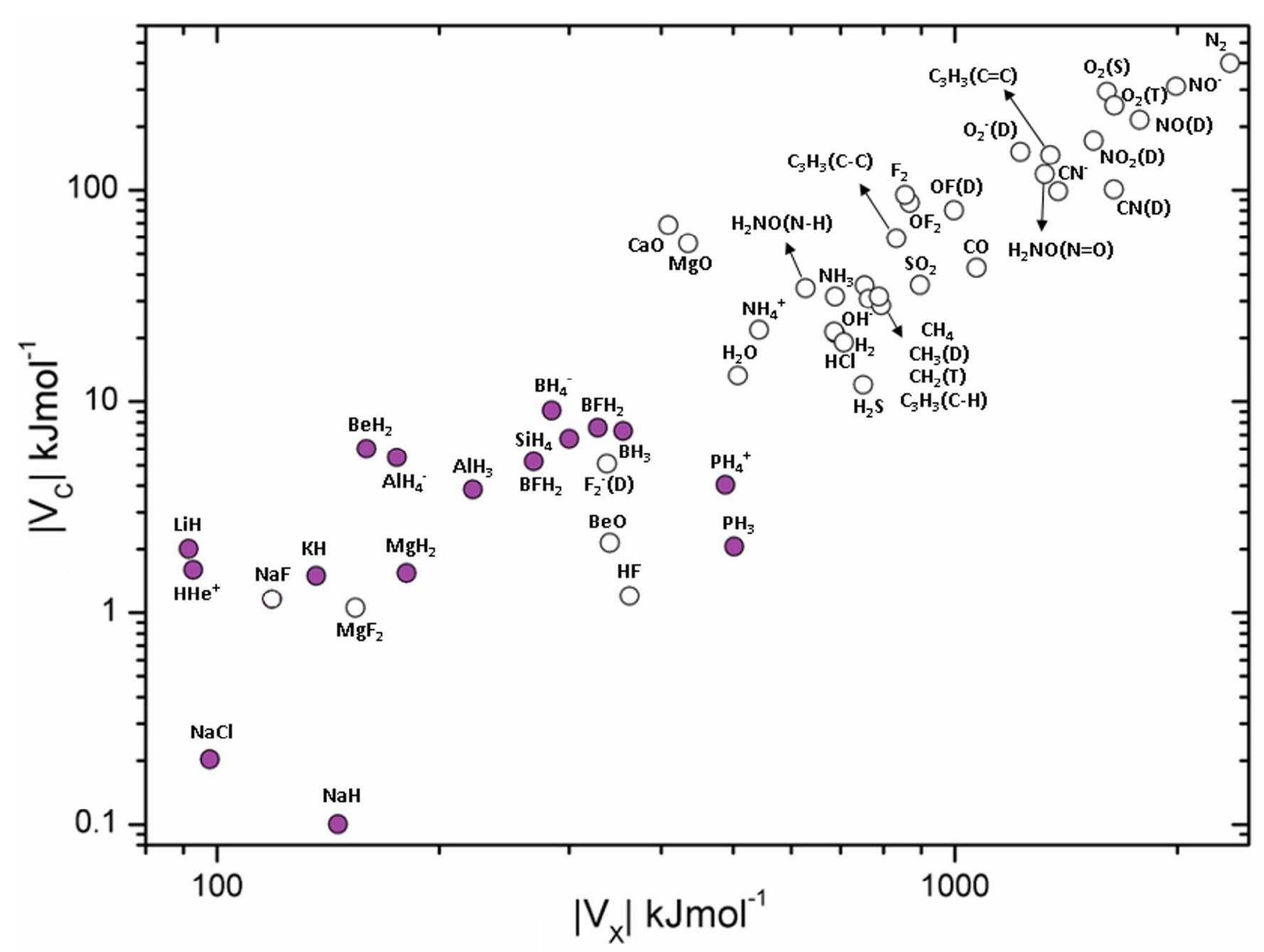

FIG. 2 Absolute value of bond correlation energy versus absolute value of bond exchange energy. Negative values for bond correlation are marked in purple. 
Figure 3a plots the absolute value of the bond correlation energy against bond length. The first point to be addressed in Fig.3a is that bond correlation can be either negative or positive. The latter part of the second row of elements has positive bond correlation energies. The crossover point between negative and positive bond correlation energies is between boron and carbon. A careful comparison between compounds containing either element

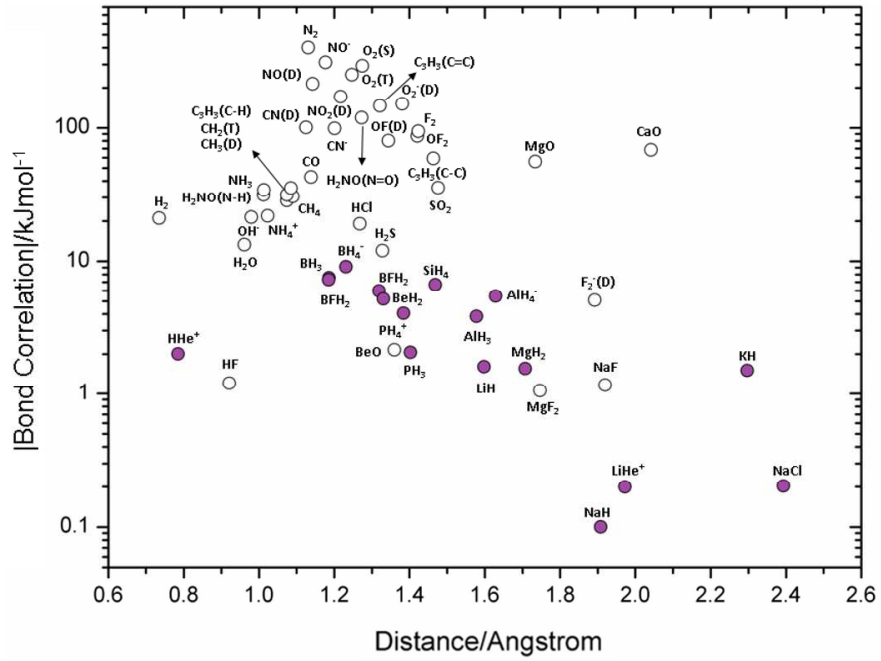

Therefore, bond order and bond polarity explain the somewhat odd position of the metallic oxides $\mathrm{MgO}$ and $\mathrm{CaO}$. The intermediary position that these molecules occupy in Fig. 3a are a consequence of the large polarity of the $\mathrm{M}=\mathrm{O}$ bond having been counteracted by the double bond.

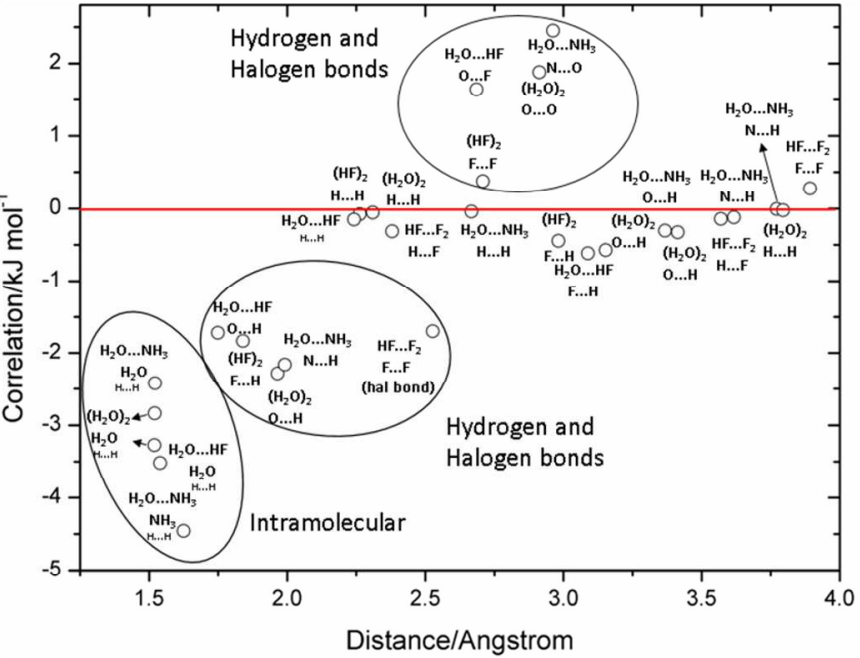

FIG 3. (a) Absolute value of the bond correlation energy against bond length. Negative values for bond correlation are marked in purple; (b) Interatomic correlation energy against internuclear distance for molecular complexes

sheds light on the nature of this cross-over. If one considers $\mathrm{BH}_{4}^{-}$, which is iso-electronic with methane and also has the same number of atoms, the bond correlation is still negative unlike that of methane. If we give $\mathrm{BH}_{4}{ }^{-}$the geometry and basis set of $\mathrm{CH}_{4}$ we still find a negative bond correlation. Moreover, it is enticing to assume that the negative bond correlation energy is a characteristic of hydrides, as all species in that group possess this property.

Figure $3 \mathrm{a}$ also reinforces how bond correlation correlates with bond polarity as the upper left quadrant of Fig. 3a is heavily populated by low polarity covalent bonds while the lower right quadrant exclusively consists of ionic species. Thus we see that homonuclear molecules, such as $\mathrm{N}_{2}$ and $\mathrm{O}_{2}$, have a very large bond correlation, and very small and even negative energies are seen in very polar or ionic bonds.

Furthermore, the extreme upper left part of Figure $3 \mathrm{a}$ is occupied by a group of molecules with unsaturated bonds. In fact, atoms bonded by two or more electron pairs exhibit bond correlation values greater than $40 \mathrm{kJmol}^{-1}$. It is clear that bond correlation energy increases with bond order.
Figure $3 \mathrm{~b}$ plots the interatomic correlation energy against internuclear distance for molecular complexes. Most points in Fig. 3b fall close to the red line, which marks zero correlation energy. However, there are three distinct clusters that stand out: (i) $\mathrm{H}$...H intramolecular interactions, (ii) hydrogen bonds (H... A, $\mathrm{A}=\mathrm{O}, \mathrm{N}, \mathrm{F})$ and a halogen bond $(\mathrm{F} \ldots \mathrm{F})$, and (iii) $\mathrm{O} \ldots \mathrm{O}, \mathrm{F} \ldots \mathrm{F}, \mathrm{O} \ldots \mathrm{N}$ and $\mathrm{F}$... O intermolecular interactions. These interatomic correlation energies are negative for the first two clusters, but positive for the third cluster. It is important to highlight that the interactions involved in the hydrogen bond (DH...A), both H...A and D...A, are as strong as the H...H intramolecular interactions in $\mathrm{H}_{2} \mathrm{O}$ and $\mathrm{NH}_{3}$. In this context one could regard the hydrogen bond as an intramolecular interaction. The third cluster also encompasses strong interactions despite of the positive values. These interactions are abnormal as they display rather large correlation energies. These interactions have been reported to be very important to the stabilization of hydrogen bonds, as observed in ref. ${ }^{24}$, pointing to the hydrogen bond as being actually a three-atom system. 
It is fruitful to introduce a measure of ionicity into the overall discussion. A simple proposal is to calculate the charge transfer between atoms according to QTAIM net atomic charges. In diatomics this measure unambiguously gauges ionic character. In general polyatomic molecules a given (net) atomic charge does not reveal how its original charge is redistributed in the molecule after its formation from the free atoms. However, symmetry in the molecule allows one to proceed. For example, in $\mathrm{MgF}_{2}$ the more electropositive magnesium has lost electronic charge to the two fluorine atoms in equal measure. Hence, one can

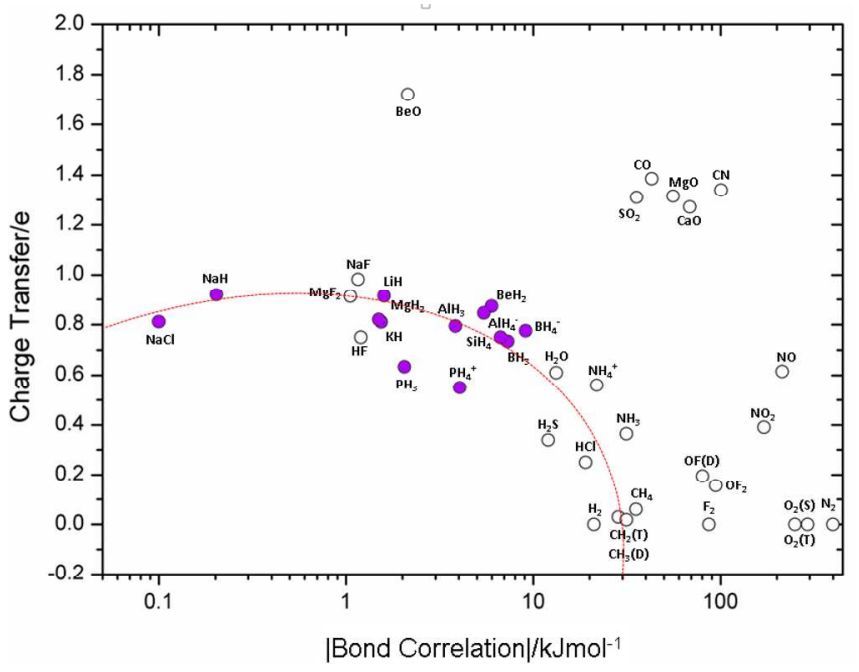

ionicity and covalency, the opposite of covalency is not ionicity. Indeed, it is perfectly possible to have a bond that is both covalent and ionic: a polar bond. A burning question is then why simple explanations of valence bond theory, and indeed a wider undergraduate education, leaves one with the impression that ionicity and covalency are opposites in a one-dimensional world.

The answer lies in the continuous red arch appearing in Fig. $4 \mathrm{~b}$ (and actually also in Fig.4a because of the strong correlation between exchange and correlation energy). The arch goes from $\mathrm{NaCl}$, an unarguably ionic molecule, to $\mathrm{F}_{2}$,

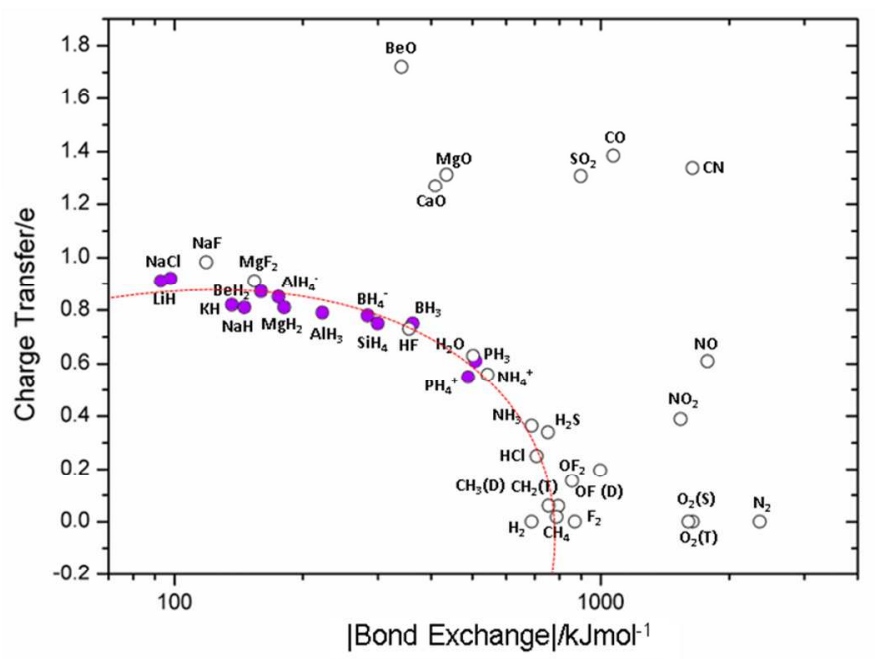

FIG 4. Charge Transfer against absolute values of (a) bond correlation and (b) bond exchange. Negative values for bond correlation are marked in purple. Note that charge transfer is defined to be always positive

unambiguously deduce the degree of charge transfer (and hence ionicity) from the atomic charges: a simple division by two of the $\mathrm{Mg}$ charge suffices in this case.

Figures $4 \mathrm{a}$ and $4 \mathrm{~b}$ plot the QTAIM charge transfer against (a) absolute values for bond correlation and (b) absolute values for bond exchange. The QTAIM charge transfers were obtained from the absolute charge on the central atom being divided by the number of ligands attached to it. The similarities between Figs. $4 \mathrm{a}$ and $4 \mathrm{~b}$ are expected given the reasonable correlation between bond correlation and exchange observed in Fig. 2. It is important to realise that bond exchange energy and charge transfer are two, in principle, independent measures. Indeed, charge transfer measures ionicity, the opposite of which is called non-ionicity, rather than covalency. Covalency is measured by bond exchange, which again presents a sliding scale from (very) covalent (high value of bond exchange energy) to non-covalent. Again, in this two-dimensional world of which is purely covalent. Near the center of this arch one finds the polar bonds. All molecules that fall outside this arch are bound by multiple bonds. In fact one could argue that these molecules form two new arches of decreasing bond polarity, one arch for double bonds and one for triple bonds.

In conclusion, we find that correlation energy is not always stabilizing in nature as is commonly asserted. For most molecules considered here, bond correlation is actually positive and therefore repulsive. The few exceptions are all hydrides in which the negative charge of the hydrogens induces a negative bond correlation. Secondly, the bond correlation energy is statistically correlated to the bond exchange energy. Thirdly, bonds can be characterized in a two-dimensional description by the independent measures of ionicity (via charge transfer) and covalency (via interatomic exchange energy). Hence, in principle, ionicity is not the opposite of covalency, unless 
one travels along a one-dimensional arc in this twodimensional world.

\section{ACKNOWLEDGMENTS}

A. F. S. thanks the Brazilian government's FAPESP for the award of postdoctoral position number 2014/21241-9 and 2015/22247-3, and P.L.A.P. acknowledges the EPSRC for funding through the award of an Established Career Fellowship (grant EP/K005472).

\section{ASSOCIATED CONTENT}

\section{Supporting Information}

The Supporting Information is available free of charge on the ACS Publications website at DOI:

(i) Complete topologically partitioned energy fingerprints (MP2-IQA) (Figures S1 to S11), (ii) methods, geometries and results of IQA correlation calculations (Tables S1 and S2), (iii) basis sets and general discussion of $\mathrm{H}_{2}, \mathrm{LiH}, \mathrm{KH}$, $\mathrm{NaH}$ and $\mathrm{BeH}_{2}$, and (iv) additional plots relating $\mathrm{V}_{\mathrm{c}}$ to $\mathrm{V}_{\mathrm{x}}$ and internuclear distance (Figures S12 to S14).

\section{AUTHOR INFORMATION}

Corresponding Author

*E-mail: pla@manchester.ac.uk.

\section{REFERENCES}

(1) Popelier, P. L. A. QCTFF: On the Construction of a Novel Protein Force Field. Int.J.Quant.Chem. 2015, 115, 1005-1011.

(2) Popelier, P. L. A. Molecular Simulation by

Knowledgeable Quantum Atoms. Phys.Scr. 2016, 91, 033007.

(3) Jeziorski, B.; Moszynski, R.; Szalewicz, K. Perturbation Theory approach to intermolecular potential energy surfaces of van der Waals complexes. Chem.Rev. 1994, 94, 1887-1930.

(4) Popelier, P. L. A. In The Nature of the Chemical Bond Revisited; Frenking, G., Shaik, S., Eds.; Wiley-VCH, Chapter 8: 2014, p 271-308.

(5) Bader, R. F. W. Atoms in Molecules. A Quantum Theory.; Oxford Univ. Press: Oxford, Great Britain, 1990.

(6) Popelier, P. L. A. Atoms in Molecules. An Introduction.; Pearson Education: London, Great Britain, 2000 .

(7) Matta, C. F.; Boyd, R. J. The Quantum Theory of Atoms in Molecules. From Solid State to DNA and Drug Design.; Wiley-VCH: Weinheim, Germany, 2007.
(8) Popelier, P. L. A.; Aicken, F. M. Atomic Properties of Amino Acids: computed Atom Types as a Guide for Future Force Field Design. ChemPhysChem 2003, 4, 824-829.

(9) Popelier, P. L. A. In The Chemical Bond - 100 years old and getting stronger; Mingos, M., Ed.; Springer: Switzerland, 2016, p 71-117.

(10) Popelier, P. L. A. In Challenges and Advances in Computational Chemistry and Physics dedicated to "Applications of Topological Methods in Molecular Chemistry"; Chauvin, R., Lepetit, C., Alikhani, E., Silvi, B., Eds.; Springer: Switzerland, 2016, p 23-52.

(11) von Lilienfeld, O. A.; Tavernelli, I.; Rothlisberger, U.; Sebatiani, D. Optimization of Effective Atom Centered Potentials for London Dispersion Forces in Density Functional Theory. Phys.Rev.Lett. 2004, 93, 153004. (12) Reilly, A. M.; Tkatchenko, A. van der Waals Dispersion Interactions in Molecular Materials: beyond Pairwise Additivity. Chemical Science 2015, 6, 3289-3301. (13) Ambrosetti, A.; Ferri, N.; DiStasio Jr., R. A.; Tkatchenko, A. Wavelike Charge Density Fluctuations and van der Waals Interactions at the Nanoscale. Science 2016, 351, 1171-1176.

(14) Grimme, S.; Antony, J.; Ehrlich, S.; Krieg, H. A Consistent and Accurate $\mathrm{Ab}$ initio Parametrization of Density Functional Dispersion Correction (DFT-D) for the 94 Elements H-Pu. J.Chem.Phys. 2010, 132, 154104154122.

(15) Tkatchenko, A.; Scheffler, M. Accurate molecular van der Waals Interaction as from Ground-state Electron Density and Free-Atom Reference Data. Phys.Rev.Lett. 2009, 102, 073005-073008.

(16) Tkatchenko, A.; DiStasio Jr, R. A.; Car, R.; Scheffler, M. Accurate and Efficient Method for Many-body van der Waals Interactions. Phys.Rev.Lett. 2012, 108, 236402.

(17) Grimme, S.; Antony, J.; Schwabe, T.; Mück-

Lichtenfeld, C. Density functional theory with dispersion corrections for supramolecular structures, aggregates, and complexes of (bio) organic molecules. Org.Biomol.Chem. 2007, 5, 741-758.

(18) Morgado, C.; Vincent, M. A.; Hillier, I. A.; Shan, X. Can the DFT-D Method the Full Range of Noncovalent Interactions Found in Large Biomolecules?

PhysChemChemPhys 2007, 9, 448-451.

(19) McDonagh, J. L.; Vincent, M. A.; Popelier, P. L. A. Partitioning Dynamic Electron Correlation Energy:

Viewing Møller-Plesset Correlation Energies through Interacting Quantum Atom (IQA) Energy Partitioning Chem.Phys.Lett. 2016, 662, 228-234.

(20) Blanco, M. A.; Martín Pendás, Á.; Francisco, E. Interacting Quantum Atoms: a Correlated Energy Decomposition Scheme Based on the Quantum Theory of Atoms in Molecules. J.Chem. Theory Comput. 2005, 1, 1096-1109.

(21) GAUSSIAN09, Revision B.01, M.J. Frisch, G. W. Trucks, H. B. Schlegel, G. E. Scuseria, M. A. Robb, J. R. 
Cheeseman, G. Scalmani, V. Barone, B. Mennucci, G. A. Petersson, H. Nakatsuji, M. Caricato, X. Li, H. P.

Hratchian, A. F. Izmaylov, J. Bloino, G. Zheng, J. L. Sonnenberg, M. Hada, M. Ehara, K. Toyota, R. Fukuda, J. Hasegawa, M. Ishida, T. Nakajima, Y. Honda, O. Kitao, H. Nakai, T. Vreven, J. A. Montgomery, Jr., J. E. Peralta, F. Ogliaro, M. Bearpark, J. J. Heyd, E. Brothers, K. N. Kudin, V. N. Staroverov, R. Kobayashi, J. Normand, K.

Raghavachari, A. Rendell, J. C. Burant, S. S. Iyengar, J. Tomasi, M. Cossi, N. Rega, J. M. Millam, M. Klene, J. E. Knox, J. B. Cross, V. Bakken, C. Adamo, J. Jaramillo, R. Gomperts, R. E. Stratmann, O. Yazyev, A. J. Austin, R. Cammi, C. Pomelli, J. W. Ochterski, R. L. Martin, K. Morokuma, V. G. Zakrzewski, G. A. Voth, P. Salvador, J. J. Dannenberg, S. Dapprich, A. D. Daniels, Ö. Farkas, J. B. Foresman, J. V. Ortiz, J. Cioslowski, and D. J. Fox, Gaussian, Inc., Wallingford CT, USA, 2009.

(22) Feynman, R. P. Forces in Molecules. Phys. Rev. 1939, 56, 340-343.

(23) English, C. A.; Venables, J. A. The Structure of the Diatomic Molecular Solids. Proc.Roy.Soc.A 1974, 340, 5780.

(24) Garcia-Revilla, M.; Francisco, E.; Popelier , P. L. A.; Martín-Pendás, Á. Domain-Averaged Exchange Correlation Energies as a Physical Underpinning for Chemical Graphs. ChemPhysChem 2013, 14, 1211-1218. 